\title{
Phase-Field Models for Simulating Physical Vapor Deposition and Grain Evolution of Isotropic Single-Phase Polycrystalline Thin Films
}

\author{
James A. Stewart ${ }^{a}$ and Douglas E. Spearot ${ }^{b, *}$ \\ ${ }^{a}$ Institute for Nanoscience \& Engineering, University of Arkansas, Fayetteville, AR 72701 USA \\ ${ }^{\mathrm{b}}$ Department of Mechanical \& Aerospace Engineering, University of Florida, Gainesville, FL 32611 USA
}

\begin{abstract}
Two models are presented based on the phase-field methodology to simulate thin film growth during physical vapor deposition (PVD), including subsurface microstructure evolution, for isotropic single-phase polycrystalline materials. The first model couples previous phase-field modeling efforts on ballistic deposition of single-phase materials and grain orientation evolution in polycrystalline materials in a sequential simulation algorithm. The second model incorporates both PVD and grain evolution dynamics into a single free energy functional for use in a phasefield model. To illustrate the capability of the proposed models in capturing combined thin film growth and subsurface grain evolution, PVD simulations of a generic single-phase polycrystalline metal are performed on substrates with different grain sizes. In both models, when the initial substrate grain sizes are smaller than the expected surface features, the thin film grains coarsen via grain boundary (GB) migration until the GBs become aligned with the valleys between the columnar surface features. Thus, each columnar feature is associated with a distinct subsurface grain, in qualitative agreement with experimental observations. Differences between the models arise when initial substrate grain sizes are larger than the surface columnar features. For example, when using the single free energy functional approach, grains contain noticeable internal low-angle variations, which are not captured using the coupled model.
\end{abstract}

Keywords:

Phase-Field Modeling, Physical Vapor Deposition, Polycrystalline Deposition, Thin Films, Microstructure Evolution, Grain Evolution

*Corresponding Author:

Email Address: dspearot@ufl.edu 


\section{Introduction}

Thin films are the focus of significant research due to their variety of applications, such as in optoelectronic and microelectronic devices, nanoelectromechanical systems, optical and protective coatings, etc. Generally, thin films have thicknesses and feature sizes in the nanoscale to microscale range and are usually grown using vapor deposition methods [1]. Here, the focus of this modeling and simulation work is on physical vapor deposition (PVD) in a vacuum environment because (i) the composition of the ejected target material is conserved during transport to the deposition substrate and (ii) the vacuum or low-pressure environment allows physical processes to dominate over gas-phase reactions and collisions during transport $[2,3]$. Therefore, chemical reactions and composition changes can be neglected in the development of a computational model for PVD. During PVD, incident atoms may not impinge on the substrate surface in a spatially and/or temporally uniform manner, thus creating fluctuations that give rise to surface roughness. These fluctuations compete with surface diffusion, which attempts to smooth the surface, resulting in nano to microscale surface features [1]. Furthermore, it is known that shadowing and re-emission effects can dominate surface diffusion and growth fluctuations by way of their nonlocal effects, leading to columnar growth. Therefore, to elucidate the fundamental dynamics of thin film growth during PVD, surface tension, diffusion, shadowing and re-emission effects must be taken into consideration [1-3].

The surface features and the details of the underlying microstructure that arise during PVD processing often influence the physical, electrical and chemical properties of the thin film. The deposition conditions and materials primarily determine the resulting surface morphology and microstructure. For example, a material with negligible mobility combined with a low substrate 
temperature or large incident vapor flux may promote growth of a disordered system [2,3]. On the other hand, many thin films are polycrystalline where the microstructure consists of nanometer to micron-sized domains that differ in crystal structure and/or grain orientation [1-7]. As such, in order to predict thin film properties, it is necessary to consider grains and grain boundaries (GBs) and their evolution during PVD [6,8], including potential connections between surface morphology and subsurface GB evolution [8,9]. Experimentally, structure zone diagrams (SZDs) have been developed to help predict the microstructures that may form as a function of deposition conditions, which are useful in determining initial deposition parameters [2]. While SZDs are useful, they are purely phenomenological and predictive only if deposition is performed using the exact same overall process. Furthermore, SZDs cannot account for or describe the underlying nanoscale mechanisms influencing microstructure evolution or the effects of multiple phase evolution and distribution on microstructure dynamics [2]. Therefore, by using modeling and simulation techniques, the aim is to develop a predictive model that contains the underlying physics necessary to describe critical aspects of PVD and microstructure evolution to provide a tool for (i) discovery studies of microstructure evolution during PVD and (ii) guiding experimentalists regarding deposition parameters for the development of advanced materials with designed properties [10].

The purpose of this work is to present two different two-dimensional (2D) modeling approaches based on the phase-field method to simulate PVD growth and subsurface grain evolution within the microstructure of an isotropic single-phase polycrystalline thin film. To the author's knowledge, there has been no attempt in the literature to address concurrent PVD and grain evolution in a comprehensive phase-field model. The phase-field method is a popular simulation 
technique based on the Ginzburg-Landau theory of phase transitions that has been successfully applied in materials science to study many complex phenomena: dendritic growth in the solidification of an undercooled liquid, solid-solid phase transitions, GB motion, pattern formation in alloys, etc. (cf. [4-6,11-14]). In the phase-field theory, a field variable is assumed to exist, which describes the phase or state of a system at every location within a model. The free energy of the system is assumed to be a functional of the field variable, thermodynamic variables, such as temperature and composition, and their gradients. Evolution of the system is then governed by Cahn-Hilliard or Allen-Cahn dynamics for conserved and non-conserved phenomena, respectively [4,5,11]. In this work, two phase-field modeling approaches are developed for simulating PVD of a single-phase polycrystalline material by leveraging previous modeling efforts on ballistic deposition of single-phase materials [15] and grain evolution in single-phase polycrystalline materials [14]. The first simulation approach couples the equations of motion for PVD and grain evolution models in a sequential simulation algorithm. The second approach incorporates the relevant energetic information for capturing PVD and grain evolution into a single free energy functional. These two models are then applied to simulate PVD of a generic single-phase polycrystalline metal on a substrate with different initial grain sizes to (i) study the influence of initial grain sizes on the evolution of a polycrystalline microstructure and (ii) illustrate and compare the utility and capability of these PVD models in capturing solid film growth and subsurface polycrystalline evolution.

\section{Previous Modeling Methodologies}

In this section, the requisite components of the equations of motion for modeling PVD and grain evolution in single-phase materials are presented. These equations of motion form the foundation 
of the phase-field models developed in this work. Full discussions of the motivating literature can be found in $[14,15]$.

\subsection{Equations of Motion for Physical Vapor Deposition}

The dynamics and morphology of a growing solid film during PVD is based on the interfacial growth model of Keblinski et al. [15]. This model was demonstrated to naturally capture prominent physical processes during PVD such as (i) arbitrary surface morphology formation, (ii) surface tension and diffusion, and (iii) nonlocal shadowing effects. To do this, two field variables were introduced. The first field variable, $f(\boldsymbol{r}, t)$, describes the growing solid where $f(\boldsymbol{r}, t) \approx 1$ defines a solid region, $f(\boldsymbol{r}, t) \approx-1$ defines a region of vacuum or no solid, and $f(\boldsymbol{r}, t) \approx 0$ naturally defines the solid-vapor interface. The second field variable, $g(\boldsymbol{r}, t) \geq 0$, describes the local density of the incident vapor, with $g(r, t)=0$ specifying a region of no incident vapor. The evolution equations of these fields are given by the following nondimensional expressions,

$$
\begin{gathered}
\frac{\partial f}{\partial t}=\boldsymbol{\nabla}^{2} \frac{\delta F_{V D}}{\delta f}+B(\boldsymbol{\nabla} f)^{2} \mathrm{~g}+C \sqrt{(\boldsymbol{\nabla} f)^{2} \mathrm{~g} \eta} \\
\frac{\partial \mathrm{g}}{\partial t}=\boldsymbol{\nabla}[D \boldsymbol{\nabla g}-\boldsymbol{A g}]-B(\boldsymbol{\nabla} f)^{2} \mathrm{~g}
\end{gathered}
$$

In Eq. (1), the first term is the Cahn-Hilliard evolution expression, which provides the model with the ability to form arbitrary surface structures and captures surface diffusion during solid growth. The second term couples the solid evolution to the vapor evolution and serves as a source term that leads to growth at the expense of the incident vapor, where $B$ is a parameter to 
modulate the growth rate. The last term provides surface fluctuations through a Gaussian distribution, $\eta(\boldsymbol{r}, t)$, where the amplitude is proportional to the square root of the aggregation rate and $C$ is a parameter to control the overall noise strength. In Eq. (2), the first term is the diffusion equation modified by the presence of an external force, $\boldsymbol{A}$. In this equation, $D$ is the diffusion coefficient and $\boldsymbol{A}$ provides a flux strength and direction to the incident vapor. The second term, which is the negative of the second term in Eq. (1), is a sink that removes vapor in regions that have been converted to solid. An additional parameter, $-1<b<0$, is also defined within this model. The purpose of this parameter is to prevent solid growth in regions away from the interface, i.e., $f(\boldsymbol{r}, t)<b$, which is physically motivated by the absence of gas-phase reactions occurring in PVD as mentioned in Section 1. The free energy functional, $F_{V D}$, is a function of $f(\boldsymbol{r}, t)$ and its gradient as shown in Eq. (3), providing a double well energy barrier between the equilibrium vacuum and solid phases. The parameter $a$ is the surface tension coefficient.

$$
F_{V D}=\int\left(-\frac{1}{2} f(\boldsymbol{r}, t)^{2}+\frac{1}{4} f(\boldsymbol{r}, t)^{4}+a(\boldsymbol{\nabla} f(\boldsymbol{r}, t))^{2}\right) d \Omega
$$

\subsection{Equations of Motion for Grain Evolution}

The evolution of subsurface grains, including GB motion and grain rotation, is based on the phase-field model of single-phase polycrystalline materials developed by Warren et al. [14]. This polycrystalline model was shown to capture grain impingement and coarsening during microstructure evolution through GB migration and grain rotation. To accomplish this, a free

energy functional is constructed with two field variables. The first field variable, $\phi(\boldsymbol{r}, t)$, distinguishes between the structurally ordered field within a grain interior $(\phi(\boldsymbol{r}, t) \approx 1)$ and 
structurally disordered regions characteristic of a liquid or vapor $(\phi(\boldsymbol{r}, t) \approx 0)$ or at a GB $(0<\phi(\boldsymbol{r}, t)<1)$. The second field variable, $\theta(\boldsymbol{r}, t)$, measures the local grain orientation from a chosen global axis (the $x$-axis in Warren et al. [14]) and varies between 0 and $2 \pi / N$, where $N$ is the rotational symmetry of the crystal. With this approach, the isotropic free energy functional is now dependent on grain misorientation and is given as,

$$
F_{P C}=\int\left(w(\phi(\boldsymbol{r}, t))+\frac{\alpha^{2}}{2}|\nabla \phi(\boldsymbol{r}, t)|^{2}+s q(\phi(\boldsymbol{r}, t))|\nabla \theta(\boldsymbol{r}, t)|+\frac{\varepsilon^{2}}{2} h(\phi(\boldsymbol{r}, t))|\nabla \theta(\boldsymbol{r}, t)|^{2}\right) d \Omega
$$

The first two terms in Eq. (4) comprise the classical components of a free energy functional. The function $w(\phi(\boldsymbol{r}, t))$ can be taken as (i) a single-well potential to model a solid polycrystalline system with grain growth occurring via GB curvature effects only (no thermodynamic driving force) or (ii) a double-well potential to model a system where a disordered phase (liquid or vapor) is allowed to coexist with the ordered polycrystalline phase and thermodynamics drive evolution. The $\nabla \phi(\boldsymbol{r}, t)$ term is the interfacial gradient energy contribution. The third and fourth terms account for grain orientation within the free energy. The third term is required for grain and GB stability while the fourth term provides the GB dynamics. The functions $q(\phi(\boldsymbol{r}, t))$ and $h(\phi(\boldsymbol{r}, t))$ are required for crystalline orientation effects to be reduced or removed in a disordered region and are both taken to be $\phi(\boldsymbol{r}, t)^{2}$. The evolution of these field variables is governed by Allen-Cahn dynamics for non-conserved systems, which gives rise to the following non-dimensional equations of motion,

$$
\tau_{\phi} \frac{\partial \phi}{\partial t}=\frac{\partial w}{\partial \phi}+\alpha^{2} \nabla^{2} \phi-2 s \phi|\nabla \theta|-\varepsilon^{2} \phi|\nabla \theta|^{2}
$$




$$
P(|\nabla \theta|) \tau_{\theta} \phi^{2} \frac{\partial \theta}{\partial t}=\nabla \cdot\left[\phi^{2}\left(\frac{s}{|\nabla \theta|}+\varepsilon^{2}\right) \nabla \theta\right]
$$

The non-dimensional coefficients $\tau_{\phi}, \tau_{\theta}, \alpha, s$, and $\varepsilon$ in Eqs. (5) and (6) are related to real thermodynamic and physical parameters, such as the latent heat of phase transformation, $L$, and a chosen size of GB features, $\varepsilon^{*}$, that correspond to the specific material and phenomena or process being modeled. First, dimensional coefficients, denoted by tildes, are determined in the following manner: $\tilde{\varepsilon}=\varepsilon^{*} \sqrt{L}, \tilde{\alpha}=1.875 \tilde{\varepsilon}$, and $\tilde{s}=1.25 a \tilde{\varepsilon}$ where $a=\sqrt{L / 2}$. Then, parameters are non-dimensionalized according to the following prescription to be used in Eqs. (5) and (6),

$$
\begin{aligned}
& (\tilde{x}, \tilde{y}, \tilde{z})=\left(l_{0} x, l_{0} x, l_{0} x\right) \quad \tilde{t}=t_{o} t \quad \tilde{\tau}_{\phi}=a^{2} t_{0} \tau_{\phi} \quad \tilde{\tau}_{\phi}=a^{2} t_{0} \tau_{\phi} \\
& \tilde{\varepsilon}=a l_{o} \varepsilon \quad \tilde{\alpha}=a l_{o} \alpha \quad \tilde{s}=a^{2} l_{o} s
\end{aligned}
$$

where $l_{o}$ and $t_{o}$ are characteristic length and time scales for the system being modeled [14]. The function $P(|\nabla \theta|)$ controls local kinetics at GBs and grain interiors, and is given as,

$$
P(|\nabla \theta|)=1-e^{-\beta \varepsilon|\nabla \theta|}+\frac{\mu}{\varepsilon} e^{-\beta \varepsilon|\nabla \theta|}
$$

where $\beta$ and $\mu$ can be chosen to increase or decrease the propensity for grain rotation and the rate of GB migration. The function $w(\phi)$ can be taken as a single-well or double-well potential depending on the physical circumstances. In the case of the single-well potential, which is most relevant and sufficient for the PVD simulations in this work, Warren et al. [14] proposed: $w(\phi)=(1 / 2)(1-\phi)^{2}$. 


\section{Approach I: Coupling PVD and Grain Evolution Models}

\subsection{Method of Coupling the Equations of Motion}

In this section, the models described in Section 2 are coupled to create a sequential phase-field simulation algorithm where, within the same time step, PVD of the thin film occurs first, followed by evolution (grain growth and rotation) of the underlying microstructure. The PVD model described in Section 2.1 is taken to control all PVD growth processes between the solid and vapor phases. Thus, this determines the allowable solid growth and evolution of the thin film at a given location. The grain evolution model described in Section 2.2 is taken to control all grain evolution below the thin film surface within the solid as defined by the PVD model. Therefore, the solid growth determined by the PVD model constrains the evolution of the polycrystalline model to occur only within those solid regions. With this approach, the features developed in the PVD model may influence grain evolution but microstructure features (e.g., grain boundaries and grain orientation) do not influence surface growth and dynamics. The impact of this unidirectional constraint will be discussed with the presentation of the simulation results.

To apply this constraint, a simple coupling approach is defined. The local solid density, $f_{d}(\boldsymbol{r}, t)$, in the PVD model is introduced as $f_{d}(\boldsymbol{r}, t)=(f(\boldsymbol{r}, t)+1) / 2$, which has values between 0 and 1 [15]. This local solid density defines the fraction of a phase-field volume that is solid (i.e., structurally ordered). Therefore, the local solid density has a similar interpretation as the $\phi(\boldsymbol{r}, t)$ field variable in the grain evolution model, which defines the fraction of the phase-field volume 
that is solid [14]. Therefore, to couple the PVD and grain evolution models in Sections 2.1 and 2.2, respectively, this work enforces the condition that $\phi(\boldsymbol{r}, t) \leq f_{d}(\boldsymbol{r}, t)$ for every phase-field volume at every time step. It should be noted that these variables cannot be taken to be strictly equal during the simulation as the $\phi(\boldsymbol{r}, t)$ variable needs to evolve freely within the solid regions, specifically decreasing to values less than $f_{d}(\boldsymbol{r}, t)$ at GBs, so GB dynamics are not artificially influenced. Therefore, the kinetic coefficient parameters, $\tau_{\phi}$ and $\tau_{\theta}$, should be carefully defined so that correlated growth of the thin film and evolution of the underlying microstructure occurs.

\subsection{Numerical Solution}

The equations of motion described in Eqs. (1), (2), (5) and (6) are discretized and solved on a uniform 100 x 100 square mesh. Eqs. (1) and (2) are solved using explicit forward first-order and centered second-order finite difference approximations for time and space, respectively. For Eqs. (1) and (2), no-flux boundary conditions are applied in the direction parallel to the substrate $(x$ axis) while fixed boundary conditions are applied perpendicular to the substrate ( $y$-axis). Eq. (5) is solved with a semi-implicit approach while Eq. (6) is solved using a fully implicit approach where the method of conjugate gradients is used to solve the associated system of equations. The discretization of Eqs (5) and (6) is given in detail in Warren et al. [14]. For Eqs. (5) and (6), noflux boundary conditions are applied at each boundary.

The initial model configuration used in this work is that of a flat substrate parallel to and spanning the $x$-axis with a thickness of 5 mesh points in the $y$-direction where $f(\boldsymbol{r}, 0)=1$ and $\mathrm{g}(\boldsymbol{r}, 0)=0$. This boundary condition is assumed constant below $y=0$ to provide a fixed 
substrate. The region above the substrate is taken to be vacuum where $f(\boldsymbol{r}, 0)=-1$ and $\mathrm{g}(\boldsymbol{r}, 0)=\mathrm{g}_{0}$. Beyond the $y=100$ boundary, this condition is held fixed to provide a continuous vapor source during deposition. Within the initial substrate, a polycrystalline microstructure is constructed where $\phi(\boldsymbol{r}, 0)=1$ and the value of $\theta(\boldsymbol{r}, 0)$ depends on the orientation ascribed to each grain within the region. Within the vacuum region $\phi(\boldsymbol{r}, 0)=0$ while $\theta(\boldsymbol{r}, 0)$ can be any arbitrary value to solve Eq. (6) as it has no meaning in this region. As such, in this work, the values of $\theta(\boldsymbol{r}, 0)$ in the vacuum region are randomly chosen from the interval $(-\pi, \pi)[14]$.

\subsection{Model Parameters}

Following Keblinski et al. [15], the non-dimensional spatial and temporal discretizations for Eqs. (1) and (2) are taken to be 1 and 0.01 , respectively. The non-dimensional model parameters in Eqs. (1) and (2) are chosen to produce porous and columnar features during simulation of purely vertical PVD providing realistic features to the thin film; these values are summarized in Table 1. The direction of growth is taken to be perpendicular to the $x$-axis, i.e., $\boldsymbol{A}=A_{y} \widehat{\boldsymbol{y}}$. The aggregation coefficient, $B$, is chosen to be large enough to convert essentially all vapor into solid at the interface. The noise coefficient, $C$, is chosen to provide sufficient noise at the surface to allow surface features to form. The diffusion coefficient, $D$, is chosen to be an order of magnitude smaller than $\boldsymbol{A}$ so the primary source of vapor transport is the flux parameter, $\boldsymbol{A}$ [15]. Finally, the density of the initial vapor field, $g_{0}$, is taken to be 1 .

Table 1. Non-dimensional parameters used to simulate columnar growth during vertical PVD.

\begin{tabular}{cccccccc}
\hline Parameter & $a$ & $b$ & $A_{y}$ & $B$ & $C$ & $D$ & $\mathrm{~g}_{0}$ \\
\hline Value & 0.5 & -0.8 & -0.6 & 10 & 2.5 & 0.01 & 1 \\
\hline
\end{tabular}


The model parameters $\varepsilon, \alpha$ and $s$ for microstructure evolution in Eqs. (5) and (6) are fixed by the choice of GB feature size, $\varepsilon^{*}$, and the latent heat for phase transformation, $L$. In this work, $\varepsilon^{*}=1$ $\mathrm{nm}$ as this is a reasonable value for GB thickness in metallic systems [16-22]. To select a reasonable value for the latent heat, it is recognized that the energetics for microstructure evolution in a metallic system are related to the melting point of the material [4]. Therefore, in this work as in Warren et al. [14], $L$ is taken to be the latent heat of fusion with a value of $2 \times 10^{9}$ $\mathrm{J} / \mathrm{m}^{3}$, which is reasonable for a metal. The non-dimensional parameters $\varepsilon, \alpha$ and $s$ in Eqs. (5) and (6) depend on a characteristic length scale, $l_{0}$, of the system being modeled and is chosen here to be $100 \mathrm{~nm}$, which is a reasonable value for the size of grains within a vapor deposited metal as they can vary from several nanometers to microns [20,21,23-25]. Using these physical quantities and the non-dimensionalization procedure in Eq. (7), the model parameters used in this work, truncated to 3 significant digits, are: $\varepsilon=0.0141, \alpha=0.0265$, and $s=0.0176$. The nondimensional spatial discretization is taken to be 0.05 , which corresponds to a dimensional value of $5 \mathrm{~nm}$ and, therefore, a physical domain size of $0.5 \mu \mathrm{m} \times 0.5 \mu \mathrm{m}$. The physical grain evolution quantities used in this work and the calculated non-dimensional quantities are in line with the values used in Warren et al. [14]. The non-dimensional temporal discretization is taken to be 0.01 while the non-dimensional parameters controlling the rate of grain growth and grain evolution kinetics are taken to be $\tau_{\phi}=1, \tau_{\theta}=1, \mu=10^{3}$, and $\beta=10^{5}$. Finally, evolution of the subsurface grains is described by the single-well potential defined in Section 2.2, which is sufficient for simulating the evolution of grains and GBs as this only occurs within the solid regions defined by the PVD model. 


\subsection{Simulation Results for Different Substrate Grain Sizes}

A series of simulations are performed to investigate the role of substrate grain size on the resulting microstructure of the vapor deposited thin film. Specifically, PVD simulations are performed on an amorphous substrate and substrates with $25 \mathrm{~nm}, 100 \mathrm{~nm}$, and $166.6 \mathrm{~nm}$ grains where the grain orientations are randomly chosen from the range $(-\pi / 2, \pi / 2)$; results are provided in Figures 1 - 4, respectively.

In Figure 1, the initially amorphous substrate coarsens into crystalline grains with distinct orientations rapidly (within 200 - 300 time steps). As the thin film grows further, grain evolution occurs via GB migration, resulting in a final microstructure with crystalline grains that contain distinct orientations and are distinguished by GB misorientations less than $\sim 10^{\circ}$. Furthermore, GBs migrate until they align with the valleys between columnar structures and grains are sufficiently large to arrest mobility. In Figure 2, PVD is simulated on a substrate with $25 \mathrm{~nm}$ grains. Again, grain coarsening occurs rapidly via grain rotation and GB migration to reduce the system's interfacial energy. As the thin film continues to grow, the grains grow to sufficient size while the GBs align themselves with the columnar structure, once again becoming immobile at the valleys between surface features. From these simulations, where the initial grain sizes are smaller than the size of the expected columnar structures, it is observed that, regardless of the initial grain size, grains will initially grow but then stagnate with a distinct orientation, at which point the grains are associated with individual columnar features. Thus, the presence of a surface structure, such as columnar features, influences grain coarsening and GB migration within the microstructure and promotes the presence of distinct grain orientations. The observation of 
columnar structures containing distinct grain orientations has been hypothesized [2,26-28] and is in qualitative agreement with experimental observations in thin film oxides [28,29], alloys [30], and aluminum [31]. Note the choice of $\tau_{\theta}$ will influence these grain evolution effects. For example, if $\tau_{\theta}$ is increased in magnitude, the grain evolution kinetics will be reduced. Similarly, if $\tau_{\theta}$ is reduced in magnitude, grain rotation and GB migration will occur at a significantly increased rate. Additional simulations have been performed with values of $\tau_{\theta}$ increased and decreased by an order of magnitude. Although grain evolution occurs at different rates, these additional simulations consistently show that GBs migrate to align with the valleys of the columnar surface features. In the case where a larger value of $\tau_{\theta}$ is used, more orientation variation is observed within each columnar grain due to slower kinetics of grain evolution within the same simulation time.

Next, shown in Figure 3, PVD simulation is performed on a substrate with $100 \mathrm{~nm}$ grains, which is slightly larger than the expected columnar structures that form. Initially, as the thin film grows, grain rotation and GB migration is negligible, indicating that the grains are sufficiently large. However, as the thin film continues to grow and surface features form, GB migration occurs with minor grain rotation so that the GBs align with the valleys between the surface features. Furthermore, the presence of a GB at the peak of a surface feature causes the formation of a new grain and GBs within the thin film microstructure. Finally, the last PVD simulation, shown in Figure 4, is performed on a substrate with $166.6 \mathrm{~nm}$ grains, which is significantly larger than the columnar features that form. Here, the initial substrate grains act as a template for the growing microstructure even with continued growth containing surface features. One of the GBs aligns with a valley between columnar features while the other GB is initially located at a peak of a 
columnar feature. The presence and evolution of this GB within the columnar feature leads to the formation of a new, smaller grain that is uniquely associated with the surface feature. As such, the presence of columnar structures influences GB migration regardless of the grain size.

\section{Approach II: A Single Free Energy Functional}

\subsection{Free Energy Functional and Equations of Motion}

In this section, a novel PVD model is developed that modifies the Keblinski et al. [15] PVD model described in Section 2.1 via the incorporation of grain orientation and GB effects in the manner proposed by Warren et al. [14] as discussed in Section 2.2. This extension enables the simulation of simultaneous solid growth, surface feature formation, grain growth, and GB migration under PVD conditions, which is an advancement on the sequential method developed in Section 3.

In the following, a physically consistent approach is outlined where the equations of motion are

determined from a single free energy functional. Using the variables $f(\boldsymbol{r}, t)$ and $\theta(\boldsymbol{r}, t)$, the proposed free energy functional is presented in Eq. (9).

$$
F=\int\left(-\frac{1}{2} f^{2}+\frac{1}{4} f^{4}+a(\nabla f)^{2}+s\left(\frac{f+1}{2}\right)^{2}|\nabla \theta|+\frac{\varepsilon^{2}}{2}\left(\frac{f+1}{2}\right)^{2}|\nabla \theta|^{2}\right) d \Omega
$$

As discussed in Section $2, f(\boldsymbol{r}, t) \approx 1$ defines a solid region and $f(\boldsymbol{r}, t) \approx-1$ defines a region of vacuum or no solid while $\theta(\boldsymbol{r}, t)$ describes the local grain orientation as measured from a defined global axis. Furthermore, the grain gradient coefficient functions (i.e., functions $q$ and $h$ 
in Eq. (4)) are taken to be quadratic in the local solid density $f_{d}(\boldsymbol{r}, t)=(f(\boldsymbol{r}, t)+1) / 2$ so that orientation effects are removed within the disordered vapor region as there is no physical meaning of order within this region.

Using this free energy functional, which contains terms to describe solid and grain evolution, all equations of motion for PVD of a single-phase polycrystalline material can then be determined. The complete set of equations used in this research is presented in Eqs. (10) - (12). Equations (10) and (11), which are the equations of motion proposed by Keblinski et al. [15], are used here to describe all PVD growth processes for the solid phase with grain influences now provided by the above free energy functional. The inclusion of the orientation parameter, $\theta(\boldsymbol{r}, t)$, requires an additional equation of motion describing its evolution. This is determined from Allen-Cahn dynamics and gives rise to the equation of motion describing grain evolution in Eq. (12).

$$
\begin{gathered}
\frac{\partial f}{\partial t}=\nabla^{2} \frac{\delta F}{\delta f}+B(\nabla f)^{2} \mathrm{~g}+C \sqrt{(\nabla f)^{2} \mathrm{~g}} \eta \\
\frac{\partial \mathrm{g}}{\partial t}=\nabla[D \nabla \mathrm{g}-\boldsymbol{A g}]-B(\boldsymbol{\nabla} f)^{2} \mathrm{~g} \\
P(|\nabla \theta|) \tau_{\theta}\left(\frac{f+1}{2}\right)^{2} \frac{\partial \theta}{\partial t}=\nabla \cdot\left[\left(\frac{f+1}{2}\right)^{2}\left(\frac{s}{|\nabla \theta|}+\varepsilon^{2}\right) \nabla \theta\right]
\end{gathered}
$$

\subsection{Numerical Solution and Model Parameters}

These equations of motion are numerically solved as in Section 3 where Eqs. (10) - (12) are discretized and solved on a uniform 100 x 100 square mesh. Eqs. (10) and (11) are solved using the explicit forward first-order and centered second-order finite difference approximations for time and space, respectively. For both of these equations, no-flux boundary conditions are 
applied in the direction parallel to the substrate ( $x$-axis) while fixed boundary conditions are applied perpendicular to the substrate ( $y$-axis). Eq. (12) is solved using a fully implicit approach where the method of conjugate gradients is implemented to solve the associated system of equations with no-flux boundary conditions applied at each boundary. The complete discretization of Eq. (12) is given in detail in Warren et al. [14].

The non-dimensional PVD model parameters in Eqs. (10) and (11) are taken to be the same as in Section 3 (provided in Table 1) to produce porous and columnar features during the simulation of purely vertical PVD. Furthermore, as Eqs. (10) - (12) are posed as purely non-dimensional expressions, the model parameters, $s$ and $\varepsilon$, controlling grain evolution in Eq. (12) are assumed to have the same values as determined in Section 3. However, the value of $\tau_{\theta}$ is taken to be an order of magnitude smaller (i.e., $\tau_{\theta}=10^{-1}$ ), thus leading to faster grain kinetics, which was found to be necessary to observe grain evolution comparable to Section 3.

\subsection{Simulation Results for Different Substrate Grain Sizes}

Using the approach outlined in Sections 4.1 and 4.2, PVD simulations are again performed on an amorphous substrate and polycrystalline substrates with $25 \mathrm{~nm}, 100 \mathrm{~nm}$, and $166.6 \mathrm{~nm}$ grains, shown in Figures 5 - 8. It should be noted that the equations of motion proposed in this section are taken to be purely non-dimensional and there is no explicit physical relationship to length scales (e.g., grain size) within these simulation. However, the results presented in this section are labeled with physical grain sizes to allow for comparison with the corresponding simulation

results presented in Section 3. From this series of PVD simulations, several qualitative observations are made for comparison with the PVD simulation results in Section 3. 
First, in Figures 5 and 6, where the initial substrate grain sizes are smaller than the surface features that form, the grains rapidly coarsen to form larger stable grains, which was also observed in Figures 1 and 2. Grain coarsening and thin film growth continues with GBs migrating to the valleys between the columnar surface features. Thus, the surface columnar features are associated with a subsurface grain. These qualitative features are consistent with the observations made in Figures 1 and 2 using the coupled simulation approach, implying that the model in Section 3 is sufficient for capturing this aspect of PVD growth. However, in Figures 5 and 6, the subsurface grains contain more noticeable orientation variations than Figures 1 and 2, caused by the random numerical noise assigned to the vapor phase, which was masked by the coupling condition used in Section 3.

Next, in Figures 7 and 8, where the initial substrate grain sizes are larger than the surface features that form, significant differences in the evolution of the polycrystalline microstructure begin to arise between the two models. In Figure 7, noticeable internal variation in grain orientation occurs during the initial stages of thin film growth, which was not observed in Figure 3 where the internal grain orientation was uniform and acted as a template for the growing microstructure. However, as thin film growth continues, the grains coarsen and reduce the internal orientation variation until they reach a sufficient size with orientations that are different than the initial substrate orientations. Additionally, the GBs again migrate to the valleys between surface columnar features, reducing the total interface length in the model. In Figure 8, again, significant internal grain orientation variation occurs during the initial stages of thin film growth. This internal orientation variation decreases as the grains continue to evolve during thin film 
growth. This leads to the formation of smaller grains within the microstructure (compared to Figure 4) with orientations that were not present in the initial substrate grains. Again, the GBs align with the valleys between surface features so that the surface features are associated with subsurface grains. From Figures 7 and 8, where the initial substrate grain sizes are larger than the surface features that form, the initial substrate grain sizes and orientations no longer act as a template to the growing microstructure, as was observed in Figures 3 and 4, and are instead influenced by the presence of surface columnar features and random orientation noise within the vapor phase.

\section{Discussion}

If the initial grain sizes are less than a critical size, both modeling approaches predict that the grains will coarsen to reduce the system energy. During this process, the grain sizes grow and become saturated near a critical size as the growing thin film becomes sufficiently thick, thus producing similar microstructures among the simulations. When using the single free energy functional approach, the grains contain internal low-angle orientation variations, which are not captured using the coupled modeling approach. These results are analogous to the conclusions of Melo et al. [32], who observed grain growth and saturation during deposition of $\mathrm{Au}$ and $\mathrm{Pt}$ on both glass and monocrystalline Si substrates.

However, when the initial substrate grains are larger than the surface columnar features, differences arise in the modeling results of subsurface grain evolution. When using the coupled modeling approach, the large initial grains provide an energetically stable microstructure and act as a template for the growing thin film. These results are analogous to Yi et al. [33] who 
performed low temperature deposition of permalloy (i.e., $\mathrm{Ni}_{0.79} \mathrm{Fe}_{0.21}$ ) onto a polycrystalline $\mathrm{Cu}$ substrate with an initial surface $\mathrm{Cu}$ layer deposited at several different temperatures, thus resulting in different average grain sizes for deposition. It was observed that the grain sizes in the surface $\mathrm{Cu}$ layers increased rapidly with increasing temperature and then stabilized in size. However, when the permalloy is deposited onto these surface $\mathrm{Cu}$ layers at the corresponding temperatures, the grain sizes in the permalloy thin film appear to slowly increase with increasing temperature until the largest grain sizes and temperatures are reached, at which point there is a rapid increase in the observed permalloy grain sizes. Furthermore, Solati et al. [34] performed deposition of Ta onto both glass and steel substrates (i.e., amorphous vs. large grained) and determined that the Ta grains on the steel substrates were $3-7$ times larger than on the glass substrates. Finally, Outemzabet et al. [35] deposited $\mathrm{Sb}$ doped $\mathrm{SnO}_{2}$ onto glass and polished $\mathrm{Si}$ substrates. Based on their SEM images, it can be determined that the grains grown on the $\mathrm{Si}$ substrate are significantly larger than on the glass substrate.

On the other hand, when using the single free energy approach with similar grain evolution parameters, there are immediate internal variations in the grain orientations, which are not predicted by the coupled modeling approach. These variations promote the formation of smaller grain sizes and different orientations than the initial substrate, which has not been reported in experiments. To further test the predictions of the single free energy approach, additional simulations are performed where the value of $s$ is increased by a factor of 10 . Recall that $s$ modulates the strength of the linear term, $|\nabla \theta|$, in Eq. (4) and (9), which is required for GBs to remain localized (i.e., stable). Keeping $\varepsilon$ constant leaves the strength of the GB dynamics term, $|\nabla \theta|^{2}$, unchanged [14]. In these simulations, the initial variations that led to the formation of 
smaller grains and different grain orientations were mitigated. As such, the simulation results predicted by the single free energy functional with the new value of $s$ are in agreement with the microstructure evolution results predicted by the coupled modeling approach and the qualitative experimental discussion above.

\section{Summary and Conclusions}

Two 2D models are presented based on the phase-field methodology for simulating PVD of a simplified single-phase polycrystalline material. The first simulation approach (Section 3) couples previous phase-field models describing PVD and grain evolution in polycrystalline materials in a sequential algorithm to allow simulation of thin film growth and subsurface grain evolution. In the second approach (Section 4), a single free energy functional is proposed that combines appropriate energetics from previous phase-field models to allow for simultaneous modeling of PVD and grain evolution in a physically consistent manner. Presenting both models illustrates which features of thin film deposition can be captured using the coupled phase-field approach compared to a more traditional single free energy functional approach. This is important, as the coupled approach is conceptually more simple to implement since evolution of the Keblinski et al. [15] deposition component of the model can be completed without considering gradients in the orientation field. In addition, the coupled approach provides a starting point for determining basic predictions that should be captured by a unified phase-field PVD model, thus aiding in the construction of the new free energy functional. The second approach developed here (i) removes the sequential coupling condition between individual phase-field models and (ii) allows both structural features predicted by the PVD to influence the evolution of the underlying microstructure, and the underlying polycrystalline microstructure 
(e.g., grains and GBs) to influence the formation and evolution of structural features predicted by the PVD model.

To illustrate the utility and capability of these models in capturing thin film growth and subsurface grain evolution, both modeling approaches are used to simulate PVD of a singlephase polycrystalline metal on substrates with various initial grain sizes. This allows for the analysis of initial substrate grain size on the evolution of a polycrystalline microstructure. Additionally, the capability and accuracy of using a coupled modeling approach as opposed to a more traditional single free energy functional approach is assessed through comparison of the simulation results. It is important to note several limitations of these models, which are assumed for the sake of simplicity. First, PVD may result in additional phase formation and/or preferred growth directions or anisotropies (cf. $[29,36]$ ), which are neglected in this initial work. Second, differences in attachment kinetics between the vapor phase and the different orientations of the thin film surface and GBs, which can influence surface diffusion and growth dynamics, are also neglected. Next, the numerical parameters for the PVD equations of motion in Equations (10) (12) are constructed to be non-dimensional and therefore are not related to experimentally specific length and time scales. To address this limitation, relationships between the kinetic and diffusion coefficients and physical quantities (i.e., interface thickness, mobility, etc.) need to be explicitly incorporated into Eqs. (10) and (11). Dimensionalization for grain evolution (i.e., Eq. (12)) can be performed in a similar manner as proposed by Warren et al. [14]. Finally, these simulations are performed only for the $2 \mathrm{D}$ case. Extending the models and simulations to $3 \mathrm{D}$ is straight forward for the PVD equations of motion, as the field variables can be evaluated over a 3D grid of pixels and the flux parameter $\boldsymbol{A}$ can be specified as a 3D vector. The grain orientation 
equation of motion would require the use of three Euler angles to describe the grain misorientations, as explained in Warren et al. [14], which would increase the computational complexity. Addressing each of these limitations will further refine the predictive capabilities of this model.

Regardless of the above simplifications and limitations, qualitative observations are made regarding the connection and interaction between thin film surface morphology and subsurface polycrystalline features (i.e., grains and GBs), which are found to have experimental justification as discussed in Section 5. In both modeling approaches, GBs tend to migrate and become immobilized at the valleys between columnar structures with these columnar structures being associated with subsurface grains regardless of the initial substrate grain sizes (cf. 2,26-31). However, when using the single free energy functional approach, the grains contain internal lowangle orientation variations, which were not captured as well using the coupled modeling approach. Ultimately, the grain evolution parameters need to be related to physical quantities for the PVD model to properly capture the underlying physics. Using the coupled phase-field modeling approach may be sufficient for determining qualitative descriptions of first order evolution processes, while a unified free energy would be required for a physically consistent description of all growth processes involved.

\section{Acknowledgments}

This research was supported by the National Science Foundation under grant CMMI \#0954505. The simulations in this work were performed at the Arkansas High Performance Computing Center (AHPCC), supported in part by the National Science Foundation through grants MRI 
\#0722625, MRI-R2 \#0959124, ARI \#0963249, \#0918970, and a grant from the Arkansas Science and Technology Authority. 


\section{Figure Captions}

Figure 1. Grain evolution ( $\theta$ field) during simulation of PVD on an amorphous substrate (random grain orientations) at time steps $=0$ (a), 2500 (b), 5000 (c), 10000 (d). The $\theta$ field is plotted for regions where $f \geq 0.5$. The color legend shows grain orientation in degrees relative to the positive $x$-axis.

Figure 2. Grain evolution ( $\theta$ field) during simulation of PVD on a polycrystalline substrate with $25 \mathrm{~nm}$ grains at time steps $=0$ (a), 2500 (b), 5000 (c), 10000 (d). The $\theta$ field is plotted for regions where $f \geq 0.5$. The color legend shows grain orientation in degrees relative to the positive $x$-axis.

Figure 3. Grain evolution ( $\theta$ field) during simulation of PVD on a polycrystalline substrate with $100 \mathrm{~nm}$ grains at time steps $=0$ (a), 2500 (b), 5000 (c), 10000 (d). The $\theta$ field is plotted for regions where $f \geq 0.5$. The color legend shows grain orientation in degrees relative to the positive $x$-axis.

Figure 4. Grain evolution ( $\theta$ field) during simulation of PVD on a polycrystalline substrate with $166.6 \mathrm{~nm}$ grains at time steps $=0$ (a), 2500 (b), 5000 (c), 10000 (d). The $\theta$ field is plotted for regions where $f \geq 0.5$. The color legend shows grain orientation in degrees relative to the positive $x$-axis.

Figure 5: Grain evolution ( $\theta$ field) during simulation of PVD on an amorphous substrate (random grain orientations) at time steps $=0$ (a), 2500 (b), 5000 (c), 10000 (d). The $\theta$ field is plotted for regions where $f \geq 0.5$. The color legend shows grain orientation in degrees relative to the positive $x$-axis.

Figure 6: Grain evolution ( $\theta$ field) during simulation of PVD on a polycrystalline substrate with $25 \mathrm{~nm}$ grains at time steps $=0$ (a), 2500 (b), 5000 (c), 10000 (d). The $\theta$ field is plotted for regions where $f \geq 0.5$. The color legend shows grain orientation in degrees relative to the positive $x$-axis.

Figure 7: Grain evolution ( $\theta$ field) during simulation of PVD on a polycrystalline substrate with $100 \mathrm{~nm}$ grains at time steps $=0$ (a), 2500 (b), 5000 (c), 10000 (d). The $\theta$ field is plotted for regions where $f \geq 0.5$. The color legend shows grain orientation in degrees relative to the positive $x$-axis.

Figure 8: Grain evolution ( $\theta$ field) during simulation of PVD on a polycrystalline substrate with $166.6 \mathrm{~nm}$ grains at time steps $=0$ (a), 2500 (b), 5000 (c), 10000 (d). The $\theta$ field is plotted for regions where $f \geq 0.5$. The color legend shows grain orientation in degrees relative to the positive $x$-axis. 


\section{References}

[1] Karabacak, T. (2011) Thin-film growth dynamics with shadowing and re-emission effects. Journal of Nanophotonics, 5, 1 - 18.

[2] Thornton, J.A. (1977) High Rate Thick Film Growth. Annual Reviews of Materials Science, 7, 239 - 260.

[3] Plummer, J.D., Deal, M. and Griffin, P.D. Silicon VLSI Technology: Fundamentals, Practice, and Modeling. Prentice Hall, 2000. Print.

[4] Boettinger, W.J., Warren, J.A., Beckermann, C. and Karma, A. (2002) Phase-Field Simulation of Solidification. Annual Review of Materials Research, 32, 163 - 194.

[5] Chen, L.Q. (2002) Phase-Field Models for Microstructure Evolution. Annual Review of Materials Research, 32, 113 - 140.

[6] Nestler, B. Garcke, H. and Stinner, B. (2005) Multicomponent alloy solidification: Phasefield modeling and simulations. Physical Review E, 71, 1 - 6.

[7] Pusztai, T. Bortel, G. and Granasy, L. (2005) Phase field modeling of polycrystalline freezing. Materials Science and Engineering A, 413 - 414, 412 - 417.

[8] Wang, X. (2015) Modeling and understanding materials microstructure evolution driven by interface energy. Computational Materials Science, 107, 1 - 7.

[9] Kobayashi, R., Warren, J.A. and Carter, W.C. (2000) A continuum model of grain boundaries. Physica D, 140, $141-150$.

[10] Simmons, J.P., Shen, C. and Wang, Y. (2000) Phase Field Modeling of Simultaneous Nucleation and Growth by Explicitly Incorporating Nucleation Events. Scripta Materialia, 43, 935 - 942.

[11] Wheeler, A.A., Boettinger, W.J. and McFadden, G.B. (1992) Phase-field modeling for isothermal phase transitions in binary alloys. Physical Review A, 45, 7424 - 7439.

[12] Steinbach, I., Pezzolla, F. and Prieler, R. (1995) Grain Selection in Faceted Crystal Growth using the Phase Field Theory. Modeling of Casting, Welding, and Advanced Solidification Processes, VII, 695 - 703.

[13] Steinbach, I., Pezzolla, F., Nestler, B., Seeßelberg, M., Prieler, R., Schmitz, G.J. and Rezende, J.L.L. (1996) A phase field concept for multiphase systems. Physica D, 94, 135 - 147. 
[14] Warren, J.A., Kobayashi, R., Lobkovsky, A.E. and Cater, W.C. (2003) Extending phase field models of solidification to polycrystalline materials. Acta Materialia, 51, 6035 6058.

[15] Keblinski, P., Maritan, A., Toigo, F., Messier, R. and Banavar, J.R. (1996) Continuum model for the growth of interfaces. Physical Review E, 53, 759 - 778.

[16] He, W., Bhole, S.D. and Chen, D. (2008) Modeling the dependence of strength on grain sizes in nanocrystalline materials. Science and Technology of Advanced Materials, 9, 015003 .

[17] Cantwell, P.R., Tang, M., Dillon, S.J., Luo, J., Rohrer, G.S. and Harmer, M.P. (2014) Grain boundary complexions. Acta Materialia, 62, 1 - 48.

[18] Herzig, C. and Divinski, S.V. (2003) Grain Boundary Diffusion in Metals: Recent Developments. Materials Transactions, 44, 14 - 27.

[19] Steyskal, E.M., Oberdorfer, B., Sprengel, W., Zehetbauer, M., Pippan, R. and Wurschum, R. (2012) Direct Experimental Determination of Grain Boundary Excess Volume in Metals. Physical Review Letters, 108, 055504.

[20] Islamgaliev, R.K., Pekala, K., Pekala, M. and Valiev, R.Z. (1997) The Determination of the Grain Boundary Width of Ultrafine Grained Copper and Nickel from Electrical Resistivity Measurements. Physica Status Solidi (A), 162, 559 - 566.

[21] Prokoshkina, D., Esin, V.A., Wilde, G. and Divinski, S.V. (2013) Grain boundary width, energy and self-diffusion in nickel: Effect of material purity. Acta Materialia, 61, 5188 5197.

[22] Spearot, D.E., Capolungo, L., Qu, J. and Cherkaoui, M. (2008) On the elastic tensile deformation of $\langle 100\rangle$ bicrystal interfaces in copper. Computational Materials Science, 42, 57 - 67.

[23] Koch, C.C., Youssef, K.M., Scattergood, R.O. and Murty, K.L. (2005) Breakthroughs in Optimization of Mechanical Properties of Nanostructured Metals and Alloys. Advanced Engineering Materials, 7, 787 - 794.

[24] McFadden, S.X., Mishra, R.S., Valiev, R.Z., Zhilyaev, A.P. and Mukherjee, A.K. (1999) Low-temperature superplasticity in nanostructured nickel and metal alloys. Nature, 398, $684-686$.

[25] Edalati, K. and Horita, Z. (2011) High-pressure torsion of pure metals: Influence of atomic bond parameters and stacking fault energy on grain size and correlation with hardness. Acta Materialia, 59, 6831 - 6836. 
[26] Petrov, I., Barna, P.B., Hultman, L. and Greene, J.E. (2003) Microstructural evolution during thin film growth. Journal of Vacuum Science and Technology A, 21, S117 - S128.

[27] Jung, Y., Sasaki, T., Tomimatsu, T., Matsunaga, K., Yamamoto, T., Kagawa, Y. and Ikuhara, Y. (2003) Distribution and structures of nanopores in YSZ-TBC deposited by EB-PVD. Science and Technology of Advanced Materials, 4, 571 - 574.

[28] Rashkova, B. (2003) Microstructural Characterization of Yttria-Stabilized Zirconia Thermal Barrier Coating Grown on Sapphire Substrates (Doctoral Dissertation). University of Stuttgart, Germany.

[29] Wada, K., Yamaguchi, N. and Matsubara, H. (2004) Crystallographic texture evolution in $\mathrm{ZrO} 2$ - Y2O3 layers produced by electron beam physical vapor deposition. Surface and Coatings Technology, 184, 55 - 62.

[30] Gandin, C., Rappaz, M., West, D. and Adams, B.L. (1995) Grain Texture Evolution during the Columnar Growth of Dendritic Alloys. Metallurgical and Materials Transactions A, 26A, 1543 - 1551.

[31] Barna, P.B. and Adamik, M. (1998) Fundamental structure forming phenomena of polycrystalline films and the structure zone models. Thin Solid Films, 317, 27 - 33.

[32] Melo, L.L., Vaz, A.R., Salvadori, M.C. and Cattani, M. (2004) Grain Sizes and Surface Roughness in Platinum and Gold Thin Films. Journal of Metastable and Nanocrystalline Materials, 20 - 21, 623 - 628.

[33] Yi. J.N., Li., X.P., Ding, J. and Seet, H.L. (2007) Study of the grain size, particle size and roughness of substrate in relation to the magnetic properties of electroplated permalloy. Journal of Alloys and Compounds, 428, 230 - 236.

[34] Solati, E. and Dorranian, D. (2013) Investigation of the Structure and Properties of Nanoscale Grain-Size $\beta$-Tantalum Thin Films. Molecular Crystals and Liquid Crystals, 571, 67 - 76 .

[35] Outemzabet, R., Bouras, N. and Kesri, N. (2007) Microstructure and physical properties of nanofaceted antimony doped tin oxide thin films deposited by chemical vapor deposition on different substrates. Thin Solid Films, 515, 6518 - 6520.

[36] Bunshah, R.F. and Juntz, R.S. (1973) Influence of Condensation Temperature on Microstructure and Tensile Properties of Titanium Sheet Produced by High-Rate Physical Vapor Deposition Process. Metallurgical Transactions, 4, 21 - 26. 


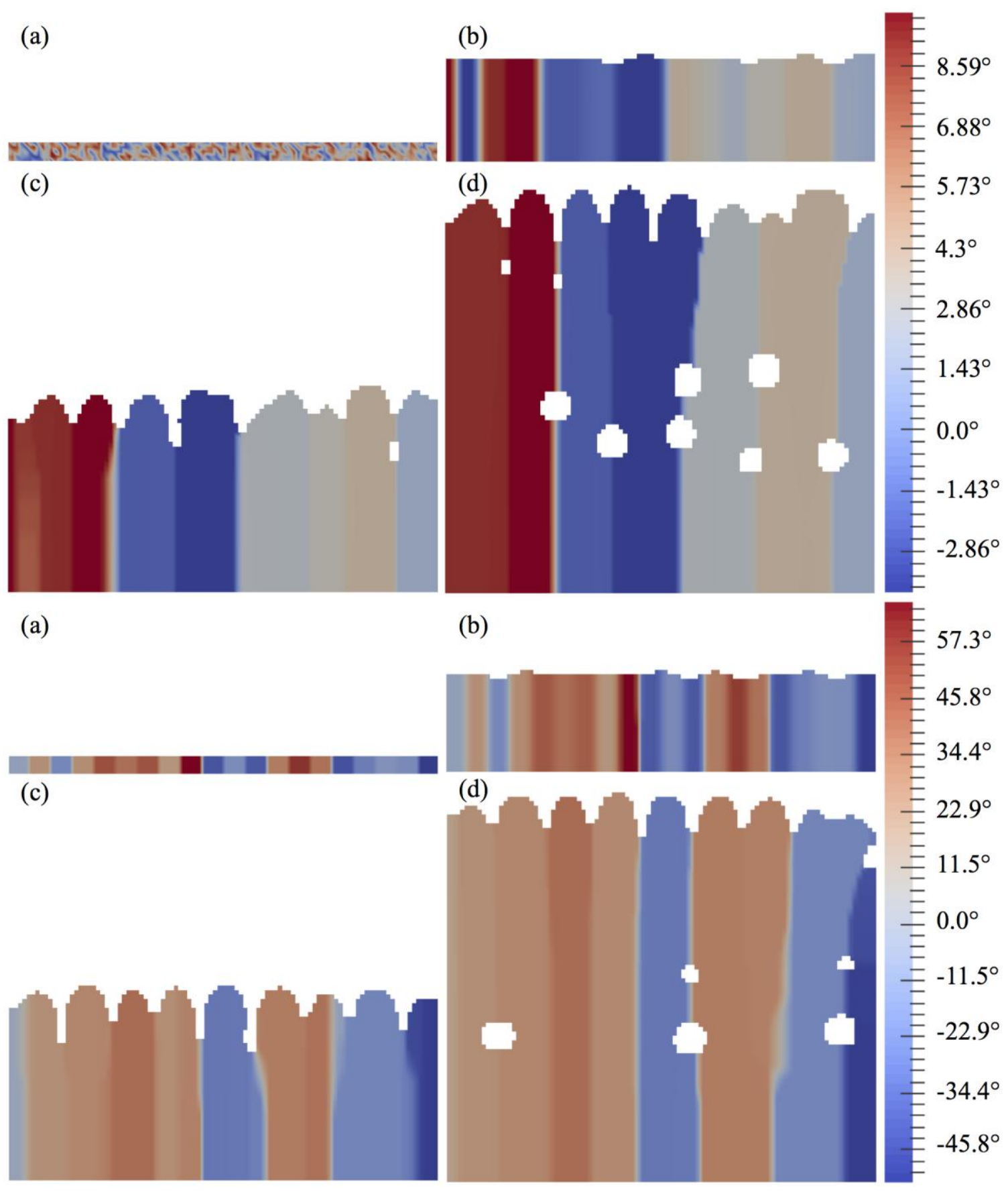




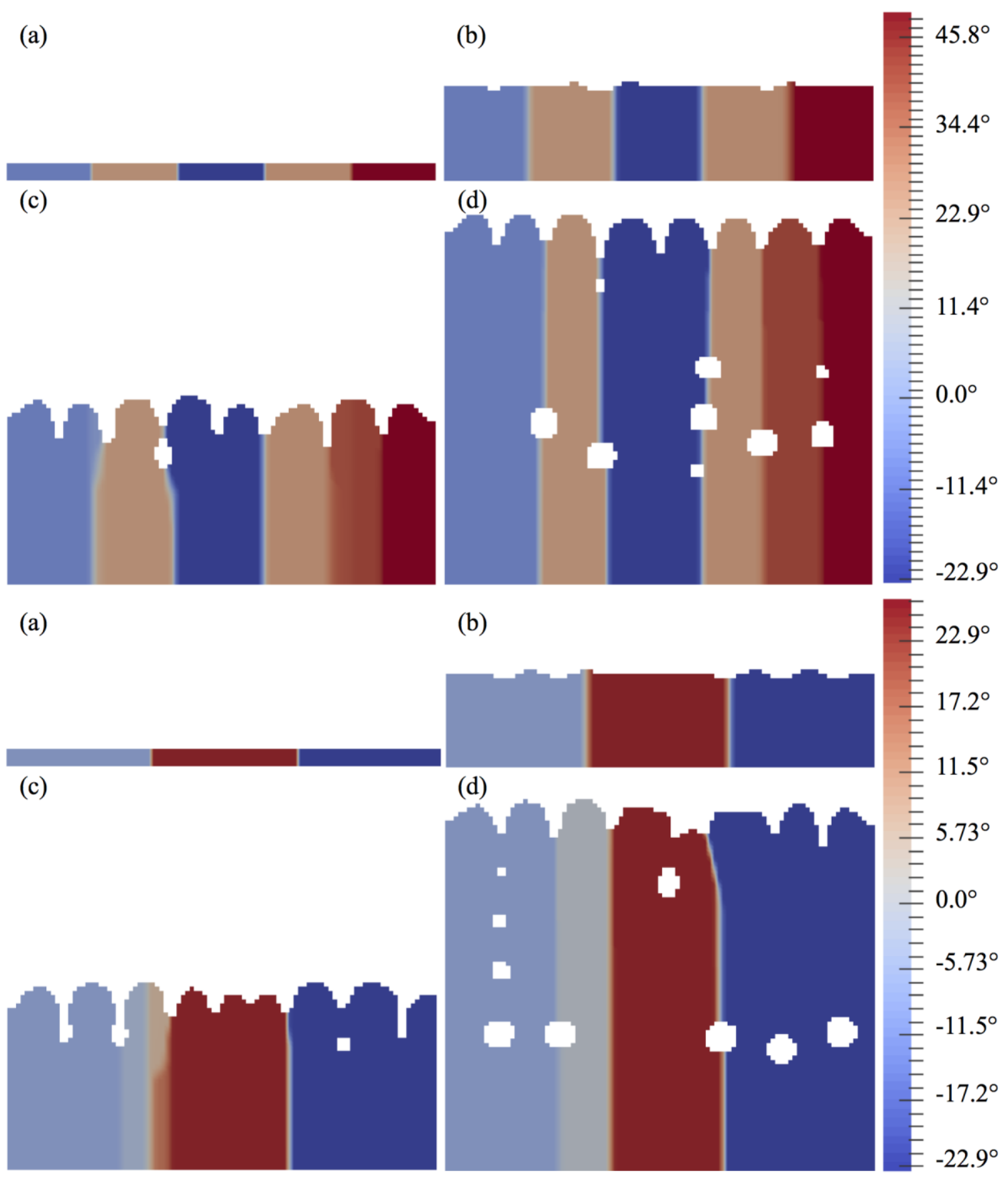




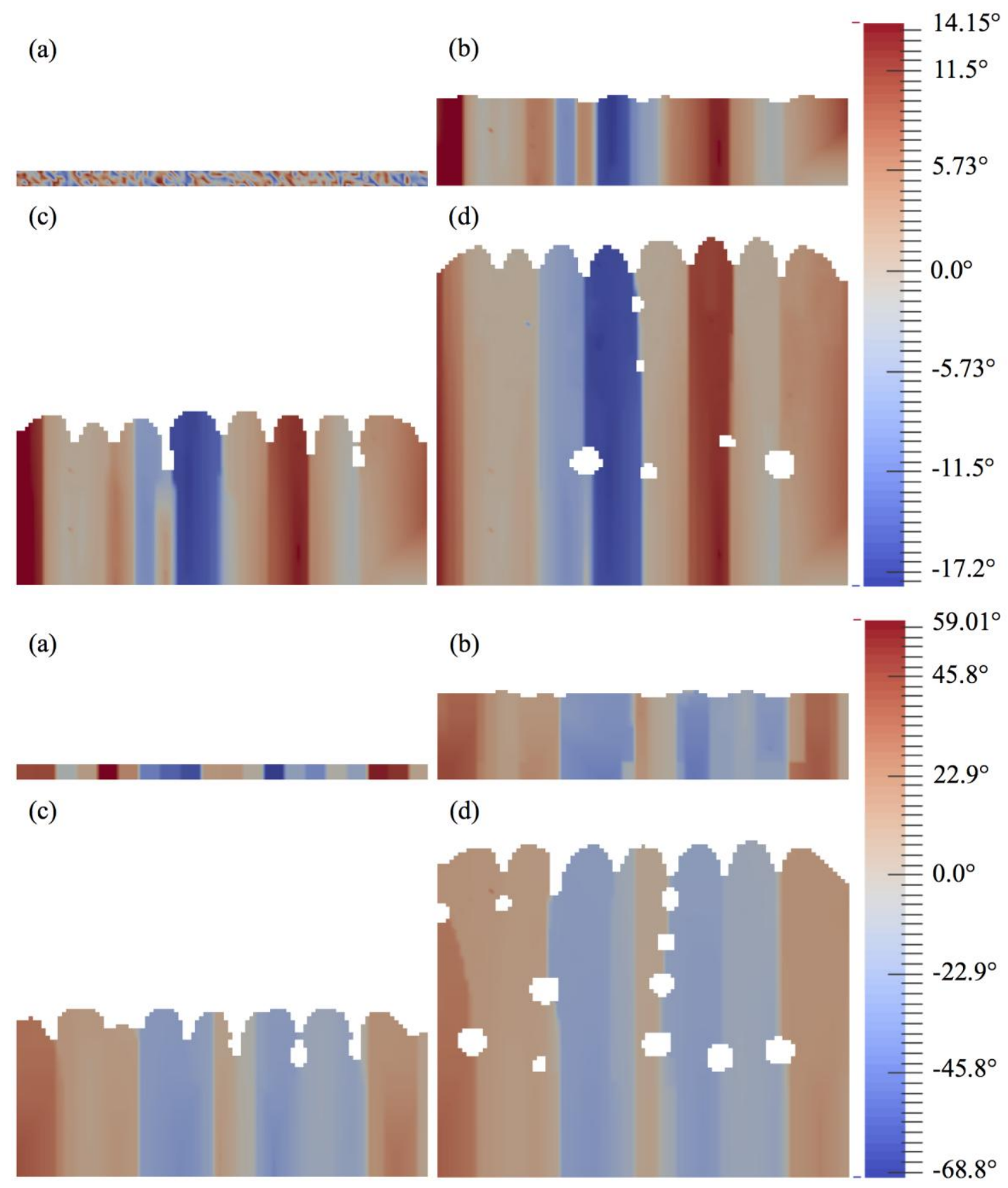




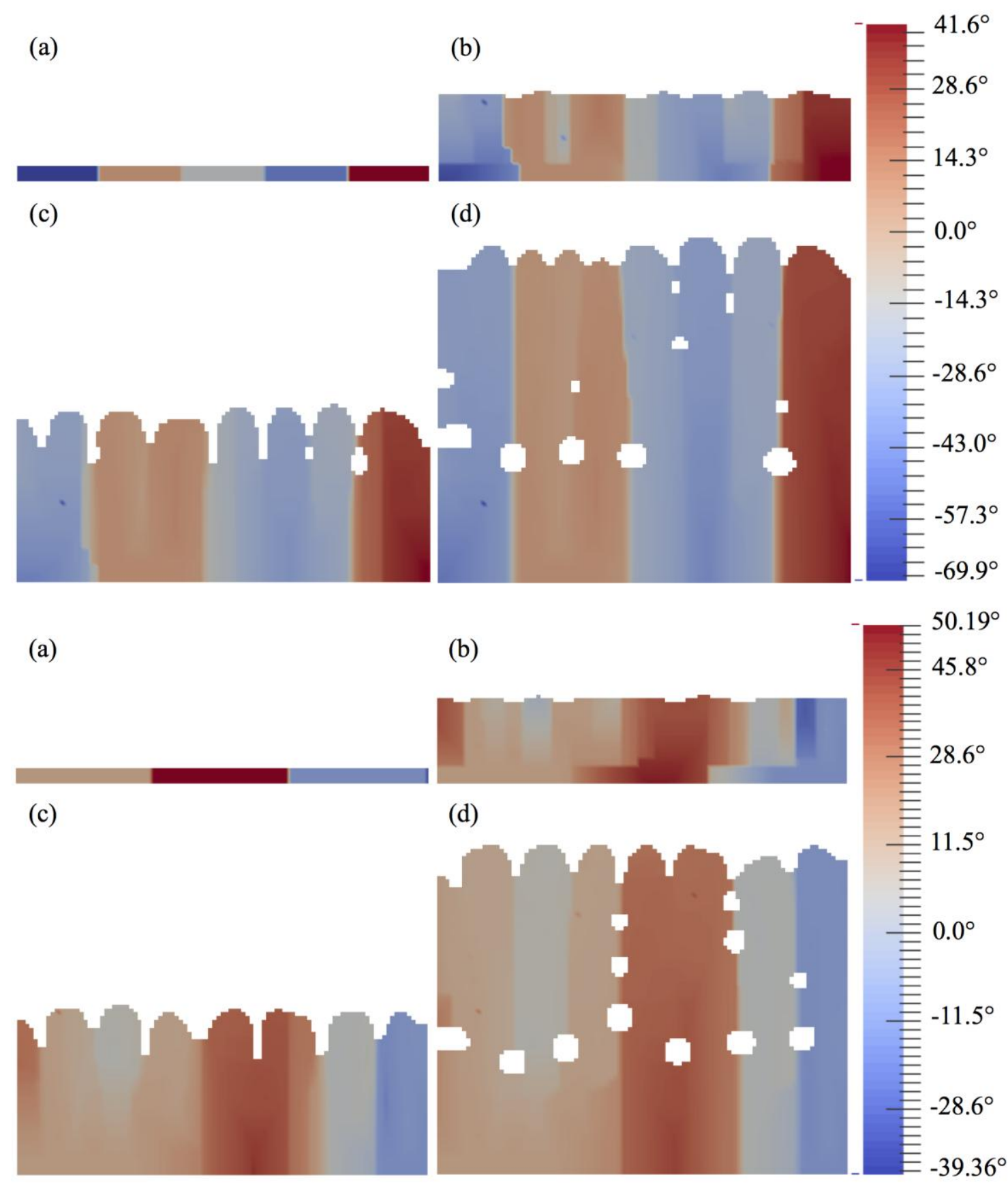


Graphical abstract

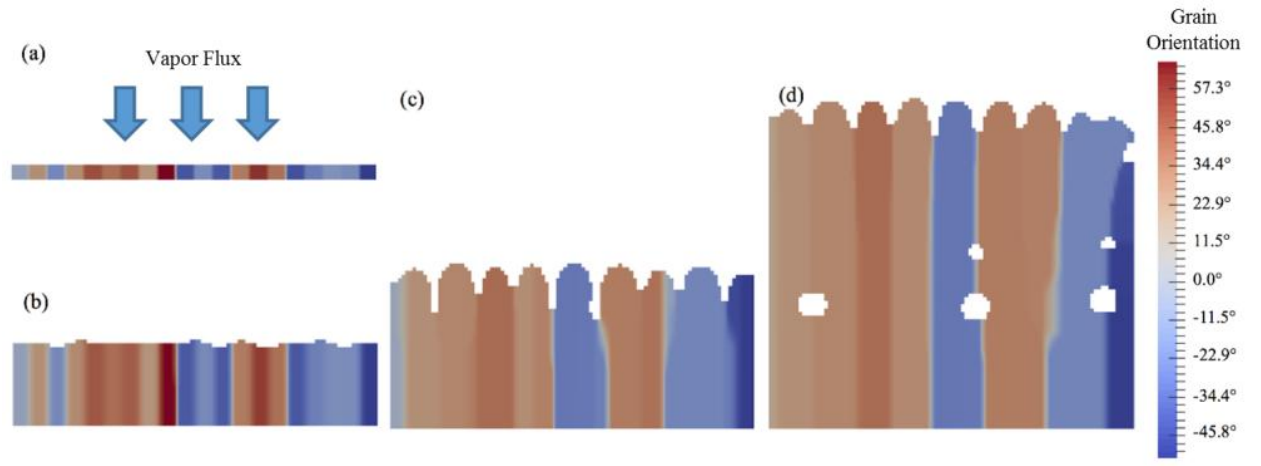

